\title{
Incidência de fungos e germinação de sementes de feijão-caupi (Vigna unguiculata L. (Walp) tratadas com óleo de nim (Azadirachta indica A. Juss)
}

SILVA, G.C.'; SANTOS,C.C.2; GOMES,D.P. ${ }^{3}$

'Bióloga, doutoranda do Programa de Microbiologia Agropecuária da Universidade Estadual Paulista - Faculdade de Ciências Agrárias e Veterinárias de Jaboticabal. E-mail:gilvaniacampos@ig.com.br. ${ }^{2}$ Geógrafa, professora da Universidade Federal do Maranhão. ${ }^{3}$ Agrônoma, doutora em Fitotecnia da Universidade Federal de Viçosa.

RESUMO: Neste trabalho avaliou-se o efeito do óleo de nim no controle de fungos associados às sementes de feijão caupi e a influência deste produto na germinação de três cultivares (Serrinha, BR 17, e Maranhão). Foram preparadas diluições de 0,5; 1,0; 2,0; 4,0 g dm 3-do óleo de nim em água destilada e testemunha, só com água. Os fungos foram identificados pelo método do papel de filtro e a germinação das sementes foi avaliada considerando as informações das Regras para Análise de Sementes. Foram utilizadas sementes de três cultivares de feijão-caupi: a cultivar Serrinha, proveniente da cidade de Timon-MA, a cultivar Maranhão, da cidade de Viana - MA, e a cultivar BR 17, obtida junto à Embrapa Meio Norte, na cidade de Teresina-PI. O crescimento de Fusarium sp. nas cultivares Maranhão e Serrinha foi reduzido em 52 e 53\%, respectivamente e o índice de redução de Aspergillus sp. foi de 14 e 20\% nas mesmas cultivares. Em relação aos fungos M. phaseolina e Phoma sp., observa-se que não foram inibidos em nenhuma das três cultivares. No que se refere à germinação das sementes nota-se que na cultivar Maranhão houve aumento no índice da germinação de 13 e 17,5\% em relação à testemunha e, na cultivar Serrinha, somente a concentração $0,5 \%$ diferiu da testemunha com redução no índice de germinação de $6,49 \%$. Conclui-se que o óleo de nim reduz a incidência de Fusarium sp. e Aspergillus sp. e é indiferente na redução de $M$. phaseolina e Phoma sp. O índice de germinação aumentou na cultivar Maranhão e diminuiu na cultivar Serrinha.

Palavras-chave: Controle de doença, Aspergillus sp., Fusarium sp., Macrophomina phaseolina, Phoma sp.

ABSTRACT: Incidence of fungi and germination of cowpea seeds (Vigna unguiculata $L$. (Walp) treated with neem oil (Azadirachta indica A. Juss). This study aimed to evaluate the effect of neem oil on germination and fungi incidence on the seeds of three cowpea cultivars (Serrinha, BR 17 and Maranhão). Dilutions of $0.5 ; 1.0 ; 2.0,4.0 \mathrm{~g} \mathrm{dm}^{-3}$ of neem oil were prepared in water. The fungi incidence was evaluated by the filter paper test, and the germination was evaluated according to the Rules for Seeds Testing ("Regras para Análise de Sementes," in Portuguese). Seeds of three cowpea cultivars were used: Serrinha and Maranhão, from the cities of Timon and Viana, respectively, state of Maranhão, Brazil, and BR 17, from Embrapa Meio Norte (Terezina, state of Piaí, Brazil). The growth of Fusarium sp. on the seed of the Maranhão and Serrinha cultivars was reduced in 52 and $53 \%$, respectively, and the reduction rate of Aspergillus sp. was 14 and $20 \%$, on the same cultivars. However, the neem oil did not inhibit the growth of the fungi Macrophomina phaseolina and Phoma sp. in any of the three cultivars. With regard to the seed germination, an increase of 13 and $17.5 \%$ was observed in the Maranhão cultivar compared to control, while for the Serrinha cultivar, only the $0.5 \%$ concentration differed from the control, reducing the germination rate by $6.49 \%$. We conclude that the neem oil was effective in controlling Aspergillus sp. and Fusarium sp. On the other hand, it was ineffective against Phoma sp. and M. phaseolina. The germination increased in the Maranhão cultivar and decreased in the Serrinha cultivar.

Keywords: Disease control, Aspergillus sp., Fusarium sp., Macrophomina phaseolina, Phoma sp. 


\section{INTRODUÇÃO}

Em todo o mundo é crescente a procura por alimentos mais nutritivos e sem substâncias tóxicas, devido à preocupação com a contaminação ambiental e dos alimentos pelo uso indiscriminado de pesticidas na agricultura. A busca por produtos naturais que sejam eficientes no controle de doenças de plantas têm aumentado nos últimos anos, visando a obtenção de alternativas aos fungicidas sintéticos e que não apresentem efeitos negativos à saúde humana e ao meio ambiente (Carneiro et al., 2007).

Estudos têm demonstrado que óleos e extratos de folhas de algumas espécies vegetais são eficientes no controle de doenças de plantas, seja pela ação fungitóxica direta ou pelo aumento no nível de resistência às doenças da cultura tratada. Estas substâncias extraídas das plantas são mais baratas que os fungicidas, facilmente disponíveis ao agricultor, apresentam baixo risco de intoxicação humana e poluição ambiental, podendo, em muitos casos, ser obtida na própria propriedade agrícola (Martinez, 2002).

No caso do nim, um grande número de compostos biologicamente ativos tem sido isolado de toda a planta, sendo assim comercialmente exploráveis. A pesquisa que envolve a planta vem crescendo significativamente, uma vez que os seus princípios ativos podem desempenhar um papel importante no manejo integrado de sistemas e na medicina alternativa. Compostos extraídos do nim se mostram seletivos, não-mutagênicos, rapidamente degradáveis, com baixa toxicidade para organismos não-alvo e benéficos, com mínimos distúrbios ao ecossistema (Mossini, 2006). Além disso, esses produtos têm a vantagem de não serem tóxicos ao homem e ser rapidamente degradados no solo (ISMAN, 2006). Os compostos extraídos do nim são considerados menos poluentes, com baixo poder residual e apresentam menor risco de intoxicação para mamíferos e aves.

O nim possui mais de 135 compostos e, segundo Quintela \& Pinheiro (2004), alguns dos principais componentes biologicamente ativos são: azadiractina, meliantriol, limoneno, odoratone e outros triterpenoides. A azadiractina, que é considerada o mais potente dos limonoides ou tetranortriterpenoides presentes no nim, causa diversas anomalias nas células (Martinez, 2002). Outros limonoides (grupo de tetranortriterpenoides), além da azadiractina, foram isolados do nim, incluindo a salanina, 14-epoxiazadiradiona, melantriol, nimbidina, nimbina, melianona, gedunina, nimbolina, ninbinem, deacetilsalanina, azadiractol, azadirona, vilosinina e meliacarpina (Kraus et al. 1987, Jones et al. 1989, Lee et al. 1991).

O feijão caupi é uma cultura de importância econômica e que é suscetível a diversas doenças, tais como a fusariose, podridão das raízes e podridão cinzenta do caule, e antracnose, causadas respectivamente pelos fungos: Fusarium sp, Macrophomina phaseolina e Colletotrichum lindemuthianum. Os fungos de armazenamento, Aspergillus $\mathrm{sp}$ e Penicillium $\mathrm{sp}$, que também contaminam as sementes, podem prejudicar a germinação, produzir toxinas e reduzir o peso seco das sementes (Torres e Bringel, 2005).

As sementes estão diretamente envolvidas na continuidade do ciclo biológico dos patógenos, de uma a outra geração do hospedeiro, e constituem o mais eficiente agente de transporte e disseminação dos patógenos, e ainda no abrigo mais seguro à sobrevivência deles.

Diante do potencial do nim em relação ao seu uso no combate ao crescimento de fitopatógenos, o objetivo do trabalho foi avaliar o efeito do óleo de nim, no controle de fungos associados às sementes de feijão caupi, e verificar a influência destes produtos na germinação das sementes.

\section{MATERIAL E MÉTODOS}

Foram utilizadas sementes de três cultivares de feijão-caupi: Serrinha, proveniente da cidade de Timon-MA, Maranhão da cidade de Viana - MA e BR 17, obtida junto à Embrapa Meio Norte na cidade de Terezina - PI.

O óleo de nim adquirido comercialmente foi diluído em água destilada na razão de 0,5 ; 1,$0 ; 2,0$ e $4,0 \mathrm{~g} \mathrm{dm}^{-3}$. As sementes de feijão-caupi foram submersas por 10 min nestas diluições e, em seguida, secas em papel filtro em temperatura ambiente. O teste de sanidade foi realizado pelo método do papel de filtro (BRASIL, 2009), com quatro repetições de 50 sementes. As sementes foram incubadas por sete dias a $20 \pm 2^{\circ} \mathrm{C}$ e fotoperíodo de $12 \mathrm{~h}$ sob luz branca fluorescente e examinadas, individualmente, sob microscópio estereoscópico para a detecção de fungos. Quando necessário, a análise foi complementada com a observação de lâminas ao microscópio ótico. O mesmo procedimento foi realizado para o controle, utilizando-se água destilada.

A germinação das sementes foi avaliada considerando-se as informações das Regras para Análise de Sementes (BRASIL, 2009). O teste padrão de germinação foi realizado empregandose, como substrato, areia previamente peneirada, lavada e esterilizada em autoclave. Foram utilizadas 4 repetições de 50 sementes, semeadas em areia, em caixas plásticas, e mantidas em condições ambiente de laboratório. A avaliação foi realizada sete dias após a semeadura, considerando apenas 
as plântulas normais como germinadas e os resultados foram expressos em porcentagem.

$\mathrm{O}$ experimento foi instalado em esquema fatorial com 5 concentrações e 3 cultivares. Os dados de porcentagem de germinação foram transformados em arco seno (x). A análise de variância e a comparação de médias foram determinadas pelos testes $\mathrm{F}$ e Tukey, respectivamente, a $5 \%$ de probabilidade.

\section{RESULTADOS E DISCUSSÃO}

Os seguintes fungos foram identificados nas sementes de feijão-caupi: Aspergillus sp., Fusarium sp., Macrophomina phaseolina e Phoma sp. O óleo de nim não afetou o crescimento de Fusarium sp. na cultivar BR 17 sendo que todas as concentrações apresentaram valores semelhantes entre si. Nas cultivares Maranhão e Serrinha observa-se que o efeito da concentração de $0,5 \%$ foi semelhante à da testemunha, não havendo redução na incidência do fungo; nas concentrações 1,0, 2,0 e 4,0\% os efeitos foram semelhantes entre si com redução média de 52 e $53 \%$ na incidência deste fungo.

Em estudos de Silva et al. (2006), realizados com óleo de nim, no controle de Fusarium oxysporum, obervou-se que as doses de 1,88 e 2,51\%, reduziu 50 e $100 \%$, respectivamente, a incidência do fungo quando comparado à testemunha. Souza et al. (2006) utilizaram diferentes concentrações de óleo de $\operatorname{nim}(0,5 ; 0,7 ; 0,9 ; 1,1 ; 1,3 \%)$ e constataram que Fusarium moniliforme apresentou sensibilidade a partir de 0,5\% e Fusarium subglutinans, a 1,3\%.

Em relação à incidência de Aspergillus sp., não foi verificada redução na cultivar BR 17; todavia, nas cultivares Maranhão e Serrinha, na concentração de 4,0\%, houve redução de 86 e $80 \%$ quando comparada a testemunha $(0,0)$. Nas demais concentrações os índices foram idênticos entre si (Tabela 2).

Ferreira et al (2006) trataram sementes de embiratanha (Pseudobombax grandiflorum (CAV).

TABELA 1. Incidência (\%) de Fusarium sp. em sementes de feijão-caupi, tratadas com óleo de nim

\begin{tabular}{|c|c|c|c|}
\hline \multicolumn{2}{|c|}{$\begin{array}{l}\text { Concentração de óleo de } \\
\text { nim (\%) }\end{array}$} & \multicolumn{2}{|l|}{ Cultivares } \\
\hline & BR 17 & Maranhão & Serrinha \\
\hline Testemunha $(0,0)$ & $6,50 \mathrm{Aa}$ & $11,00 \mathrm{Ab}$ & $11,75 \mathrm{Ab}$ \\
\hline 0,5 & $5,00 \mathrm{Aa}$ & $10,00 \mathrm{Ab}$ & $11,00 \mathrm{Ab}$ \\
\hline 1,0 & $4,75 \mathrm{Aa}$ & $8,25 \mathrm{Aa}$ & $7,25 \mathrm{Aa}$ \\
\hline 2,0 & $4,50 \mathrm{Aa}$ & $5,75 \mathrm{Aa}$ & $6,00 \mathrm{Aa}$ \\
\hline 4,0 & $2,75 \mathrm{Aa}$ & $3,25 \mathrm{Aa}$ & $5,75 \mathrm{Aa}$ \\
\hline CV & 3,25 & 8,75 & 6,0 \\
\hline
\end{tabular}

Médias seguidas de mesma letra maiúscula nas linhas e minúscula, nas colunas, não diferem pelo teste de Tukey

TABELA 2. Incidência (\%) Aspergillus sp. em sementes de feijão-caupi, tratadas com óleo de nim.

\begin{tabular}{llll}
\hline Concentração de óleo de nim (\%) & \multicolumn{3}{l}{ Cultivares } \\
\hline & BR 17 & Maranhão & Serrinha \\
\hline Testemunha $(0,0)$ & $25,00 \mathrm{Aa}$ & $11,75 \mathrm{Aa}$ & $11,25 \mathrm{Aa}$ \\
0,5 & $20,75 \mathrm{ABa}$ & $10,00 \mathrm{Aa}$ & $10,00 \mathrm{Aa}$ \\
1,0 & $18,50 \mathrm{ABa}$ & $5,50 \mathrm{ABb}$ & $9,25 \mathrm{Aa}$ \\
2,0 & $16,50 \mathrm{ABa}$ & $3,50 \mathrm{ABb}$ & $5,00 \mathrm{Bb}$ \\
4,0 & $13,50 \mathrm{Ba}$ & $1,75 \mathrm{Bc}$ & $2,25 \mathrm{Bc}$ \\
\hline CV & $\mathbf{1 1 , 5 0}$ & $\mathbf{1 0 , 0}$ & $\mathbf{9 , 0}$
\end{tabular}

Médias seguidas de mesma letra maiúscula nas linhas e minúscula, nas colunas, não diferem pelo teste de Tukey 
A. Rob.), com extrato de nim a 50 e $100 \mathrm{~g} \mathrm{dm}^{-3} \mathrm{de}$ extrato de nim, e obtiveram redução de 30 e 27 \% na redução de Aspergillus niger em relação às sementes não tratadas. Campos (2010) constatou que em sementes de algodão, o extrato da folha de nim não afetou o crescimento de Aspergillus flavus, porém bloqueou a produção de aflatoxina.

No presente trabalho, o óleo de nim não foi capaz de inibir o crescimento de M. phaseolina em nenhuma das três cultivares (Tabela 3 ).

Resultados semelhantes foram relatados por Silva et al. (2011), que ao avaliarem a influência do extrato das folhas de nim sobre o crescimento de Macrophomina phaseolina em feijão caupi, também observaram que não houve alteração na incidência do patógeno nas cultivares Maranhão e BR 17. Entretanto, na cultivar Serrinha, os autores verificaram redução de cerca de $68 \%$ na incidência do fungo nas diferentes concentrações $(0,5 ; 1,0 ; 2,0$; $\left.4,0 \mathrm{~g} \mathrm{dm}^{-1}\right)$ em relação à testemunha $(0,0)$. Bhutta et al. (2001), utilizando o extrato de sementes de nim a $0,5 \%$ e $1 \%$ in vitro, observaram a inibição do crescimento de Macrophomina phaseolina e
Alternaria alternata.

No que se refere à incidência do gênero Phoma, os resultados foram semelhantes aos de M. phaseolina, sendo que não houve interferência do óleo em nenhuma das concentrações nas três cultivares analisadas, conforme tabela 4.

Pignoni \& Carneiro (2005) relataram também uma baixa eficiência do nim no controle da antracnose e da pinta preta. Os autores atribuem tal fato às condições ambientais e à pressão do inóculo serem extremamente favoráveis ao desenvolvimento dos sintomas das duas doenças, à decomposição da azadiractina com o passar do tempo, e à pequena ação dos compostos ativos do nim sobre os fungos estudados. Outro fator que pode ter influenciado no fraco desempenho do óleo de nim no combate ao crescimento dos fungos é que a azadirachtina pode decompor-se com o tempo (Martinez 2002). Devese considerar também que, provavelmente, o nim apresenta maior eficácia contra alguns fitopatógenos do que contra outros ao mesmo tempo em que alguns patógenos são mais resistentes que outros.

No que se refere à germinação das

TABELA 3. Incidência (\%) de M. phaseolina em sementes de feijão-caupi, tratadas com óleo de nim

\begin{tabular}{llll}
\hline Concentração de óleo de nim (\%) & \multicolumn{3}{c}{ Cultivares } \\
\hline Testemunha $(0,0)$ & BR 17 & Maranhão & Serrinha \\
0,5 & $20,00 \mathrm{Aa}$ & $12,25 \mathrm{Ab}$ & $11,50 \mathrm{Ab}$ \\
1,0 & $20,00 \mathrm{Aa}$ & $11,25 \mathrm{Ab}$ & $11,00 \mathrm{Ab}$ \\
2,0 & $20,00 \mathrm{Aa}$ & $11,25 \mathrm{Ab}$ & $10,00 \mathrm{Ab}$ \\
4,0 & $20,00 \mathrm{Aa}$ & $10,00 \mathrm{Ab}$ & $7,50 \mathrm{Ab}$ \\
\hline $\mathbf{C V}$ & $20,00 \mathrm{Aa}$ & $10,00 \mathrm{Ab}$ & $7,00 \mathrm{Ab}$ \\
\hline
\end{tabular}

Médias seguidas de mesma letra maiúscula nas linhas e minúscula nas colunas, não diferem pelo teste de Tukey

TABELA 4. Incidência (\%) de Phoma sp em sementes de feijão-caupi, tratadas com óleo de nim

\begin{tabular}{|c|c|c|c|}
\hline \multirow[t]{2}{*}{ Concentração de óleo de nim (\%) } & \multicolumn{3}{|c|}{ Cultivares } \\
\hline & BR 17 & Maranhão & Serrinha \\
\hline Testemunha $(0,0)$ & $30,00 \mathrm{Aa}$ & $4,50 \mathrm{Ab}$ & $25,00 \mathrm{Aa}$ \\
\hline 0,5 & $30,00 \mathrm{Aa}$ & $2,75 \mathrm{Ab}$ & $25,00 \mathrm{Aa}$ \\
\hline 1,0 & $30,00 \mathrm{Aa}$ & $2,50 \mathrm{Ab}$ & $25,00 \mathrm{Aa}$ \\
\hline 2,0 & $30,00 \mathrm{Aa}$ & $1,50 \mathrm{Ab}$ & $25,00 \mathrm{Aa}$ \\
\hline 4,0 & $30,00 \mathrm{Aa}$ & $1,25 \mathrm{Ab}$ & $25,00 \mathrm{Aa}$ \\
\hline CV & 0,0 & 3,25 & 0,0 \\
\hline
\end{tabular}


TABELA 5. efeito de óleo de nim, sobre a germinação de sementes de feijão-caupi

\begin{tabular}{llll}
\hline Concentração de óleo de nim (\%) & \multicolumn{3}{l}{ Cultivares } \\
\hline & BR 17 & Maranhão & Serrinha \\
\hline Testemunha $(0,0)$ & $44,41 \mathrm{Aa}$ & $35,08 \mathrm{Bb}$ & $50,56 \mathrm{Aa}$ \\
0,5 & $46,74 \mathrm{Aa}$ & $32,75 \mathrm{Bb}$ & $32,84 \mathrm{Bb}$ \\
1,0 & $43,36 \mathrm{Aa}$ & $42,66 \mathrm{Aa}$ & $39,19 \mathrm{Ba}$ \\
2,0 & $41,25 \mathrm{Aa}$ & $53,75 \mathrm{Aa}$ & $36,26 \mathrm{Bb}$ \\
4,0 & $42,25 \mathrm{Ab}$ & $61,47 \mathrm{Aa}$ & $41,24 \mathrm{Ab}$ \\
\hline CV & $\mathbf{2 , 1 5}$ & $\mathbf{2 5 , 0}$ & $\mathbf{9 , 2 3}$ \\
\hline
\end{tabular}

Médias seguidas de mesma letra maiúscula nas linhas e minúscula, nas colunas, não diferem pelo teste de Tukey

sementes, na cultivar B17, não houve diferença significativa entre as quatro concentrações e a testemunha (TABELA 5). Na cultivar Maranhão, as concentrações de 0,5 e 1,0\% foram idênticas à testemunha, porém, não interferiram na germinação das sementes. Por outro lado, as concentrações 2,0 e $4,0 \%$ foram iguais entre si, mas diferiram da testemunha, com aumento na germinação de 15,3 e $17,5 \%$, em relação à testemunha. Na cultivar Serrinha, somente a concentração $0,5 \%$ diferiu da testemunha com redução no índice de germinação de $6,49 \%$ (TABELA 5).

Silva et al. (2007) observaram que o extrato de nim não afetou a germinação das sementes Phaseolus vulgaris, nem o crescimento das raízes primárias das plântulas. Xuan et al.(2004) demonstraram, em experimentos de laboratório e no solo, o efeito alelopático proveniente de compostos fenólicos presentes na casca e folhas do nim sobre a germinação e o crescimento de alfafa (Medicago sativa L.), feijão (Vigna angularis), cenoura (Daucus carota L.), rabanete (Raphanus sativus L.), arroz (Oryza sativa L.), sesame (Sesamum indicum L.), e ervas-daninhas Echinochloa crus-galli, Monochoria vaginalis, e Aeschynomene indica $\mathrm{L}$.

Conclui-se que o óleo de nim foi eficaz na redução da incidência de Fusarium sp. e Aspergillus sp. nas cultivares Maranhão e Serrinha. Entretanto, não foi eficaz na redução da incidência de Macrophomina phaseolina e Phoma sp. em nenhuma das cultivares. Quanto ao índice de germinação, o tratamento com óleo de nim aumentou na cultivar Maranhão (nas concentrações 2 e $4 \%$ ) e diminuiu na cultivar Serrinha (apenas na concentração $0,5 \%$ ).

\section{REFERÊNCIA}

BHUTTA, A.R.; BHATTI, M.H.R.; IFTIKHAR, A. Effect of seed diffusates on fungal population and germination of sunflower seeds. Hélia, Novi Sad, v.24 n.34, p.7781, 2001.
BRASIL. Ministério da Agricultura. Secretaria Nacional de Defesa Agropecuária. Regras para análise de sementes. Brasília: Ministério da Agricultura, Pecuária e Abastecimento, 2009. 395p.

CAMPOS, S. Nim - inseticida orgânico. Meio Ambiente/Ecologia. Disponível em <http://www. drashirleydecampos.com.br/categorias/53/>. Acesso em: 06 out. 2010.

CARNEIRO, S. M.T.P.G.; Pignoni, E.;VASCONCELOS, M.E.C.; GOMES, J. C. Eficácia de extratos de nim para o controle do oídio do feijoeiro. Summa phytopathologica. vol.33 no.1 Botucatu Jan./Mar. 2007. FERREIRA, K. S. C.; SANTOS, G. J. C; SILVA, C. C. Avaliação do tratamento com extrato de nim na redução da incidência de Aspergillus niger assocoados às sementes de embiratanha. Fitopatologia brasileira, v.31 (suplemento), p.312, 2006.

ISMAN, M.B. Botanical insecticides, deterrents, and repellents in modern agriculture and an increasingly regulated world. Annual Review of Entomology, v.51, pp.45-66. 2006.

JONES, P.S. S.V. LEY, E.D. MORGAN \& D. SANTAFIANOS. 1989. The chemistry of the neem tree, p. 47-67. In M. Jacobson (ed.), Focus on phytochemical pesticides. (National Research Council 1992) vol. 1, The neem tree. CRC, Boca Raton, 178p.

KRAUS, W., M. BOKEL, A. BRUHN, R. CRAMER, I. KLAIBER, A. KLAIBER, A. KLENK, G. NAGL, H. POHNL, H. SADLO \& B. VOGLER.. Structure determination by $\mathrm{nmr}$ of azadirachtin and related compounds from Azadirachta indica A. Juss (Meliaceae). Tetrah. Lett. v. 43, pp. 2817-2830. 1987.

LEE, S.M., J.A. KLOCKE, M.A. BARNBY, R.B. YAMASAKI \& M.F. BALANDRIN. 1991. Insecticidal constituents of Azadirachta indica and Melia azedarach (Meliaceae). ACS Symp. Series. v. 449, pp. 293-304. 1991.

MARTINEZ, S. S. (Ed.). O nim Azadirachta indica: natureza, usos múltiplos, produção. Londrina: Instituto Agronômico do Paraná, 2002. 142 p.

MOSSINI, S.A.G.; KEMMELMEIER, C. A árvore Nim (Azadirachta indica. A. Juss.): múltiplos usos. Acta Farmaceutica Bonaerense, v.24, n.1, p.139-148, 2005.

PIGNONI, E.; CARNEIRO, S.M.T.P.G. Severidade da antracnose em feijoeiro e pinta preta em tomateiro sob diferentes concentrações de óleo de nim em casa de vegetação. Revista Brasileira de Plantas Medicinais.

Rev. Bras. PI. Med., Campinas, v.16, n.4, p.850-855, 2014. 
Botucatu, v.8, n.1, p.68-72, 2005.

QUINTELA, E. D. \& PINHEIRO, P.V. 2004. Efeito de extratos botânicos sobre a oviposição de Bemisia tabaci biótipo B em feijoeiro. Comunicado Técnico 92, Embrapa Arroz e Feijão, Santo Antônio de Goiás, GO. $6 p$.

SILVA, G. C.; GOMES, D. P.; SANTOS, C. C. Sementes de feijão-caupi (Vigna unguiculata I. (walp), tratadas com extrato de folhas de nim (azadirachta indica a. juss.) avaliação da germinação e da incidência de fungos. Scientia Agrária. V.12,n.1,2011.

SILVA, L. S.; XAVIER, A. A.; RIBEIRO, R. C. F.; GOMES, L. I. S.; NIESCTHE, S.; MIZOBUSTSI, E. H.; SANTOS, T. M.; SILVEIRA, E. K. C. P. utilização do óleo de nim adicionado via água de irrigação no manejo do mal do panamá. Revista Brasileira de Fitopatologia. Vol. 31 - Suplemento. ISSN 0100-4158, 2006.

SILVA, J. P.; CROTTI, A. E. M.; CUNHA, W. Antifeedant and allelopathic activities of the hydroalcoholic extract obtained from Neem (Azadirachta indica) leaves. Brazilian Journal of Pharmacognosy, v. 17, n. 4, p. 529-532, 2007.

SOUZA, A. D.; CAMILO, S. B.; CAMPACCI, M. H.; MOZZER, K. C. O.; SANCHES, A. \& FURLAN, M. R. Efeito de produtos alternativos sobre o crescimento micelial de Penicilium sp, Fusarium moniliforme e Fusarium subglutinans in vitro. Summa Phytopathologica. Vol 32 - Suplemento. Issn 01005405, 2006.

TORRES, S.B.; BRINGEL, J.M.M. Avaliação da qualidade sanitária e fisiológica de sementes de feijãomacassar. Caatinga, Mossoró, v.18, n.2, p.88-92, 2005.

XUAN, T.D.; T. EIJI, T. HIROYUKI; M. MITSUHIRO; T.D. KHANH; I.M. CHUNG. Assessment of phytotoxic action of Ageratum conyzoides L. (billy goat weed) on weeds. Crop Protection .23: 335-45, 2004. 